\title{
Figure S2
}

A

Exon 16

$\begin{array}{llllllll}\mathbf{Y} & \mathbf{R} & \mathrm{A} & \mathrm{N} & \mathbf{T} & \mathrm{Y} & \mathrm{C} & \mathrm{S}\end{array}$

Mmu ... agATACCGAGCCAACACCTACTGCTCC ...

Hgla . . agCTGTTGAGCCAACACCTATTGCTCC . . .

Fdam . . agTTATCGAGCCAACACCTACTGCTCC . . .

$$
\begin{array}{llllllll}
\mathbf{Y} & \mathbf{R} & \mathrm{A} & \mathrm{N} & \mathbf{T} & \mathrm{Y} & \mathrm{C} & \mathrm{S}
\end{array}
$$

Exon 20

$$
\begin{array}{lllllllllll}
\mathbf{N} & \mathbf{L} & \mathbf{R} & \mathbf{W} & \mathbf{M} & \mathbf{A} & \mathbf{P} & \mathbf{E} & \mathbf{V} & \mathbf{F} & \mathbf{T}
\end{array}
$$

Mmu . . agAACCTGCGCTGGATGGCCCCCGAGGTGTTCACA. . .

Hgla . . agAACCTGCGCTGGATGGCCCGGG-GGTGTTCACG

Fdam . . agAACCTGGGCTGGATGGCGCCGGAGGCGTTCACA . . .

$$
\begin{array}{lllllllllll}
\mathbf{N} & \text { L } & \text { G } & \text { W } & \text { M } & \text { A } & \text { P } & \text { E } & \text { A } & \text { F } & \text { T }
\end{array}
$$

Exon 21

$$
\begin{array}{llllllllllll}
I & S & S & \text { L } & \text { L } & M & R & G & \text { W } & \text { N } & A & C
\end{array}
$$

Mmu . . ATCTCATCTCTGCTGATGCGGGGCTGGAATGCATGT . . .

Hgla . . . АTATCCTCTCTGCTGATGTGAGGTTGGAATGCGTGT . . .

Fdam. . . АTATCTTCTCTGCTTATGCAATGTTGGAATGTATGT . . .

$$
\begin{array}{lllllllllllll}
I & S & S & \text { L } & \text { L } & M & Q & C & \text { W } & \text { N } & \text { V } & \text { C }
\end{array}
$$

B

H. glaber exon 20

A G A A C C T G C G C T G G A T G G C C C G G G G G T G T T C A C G

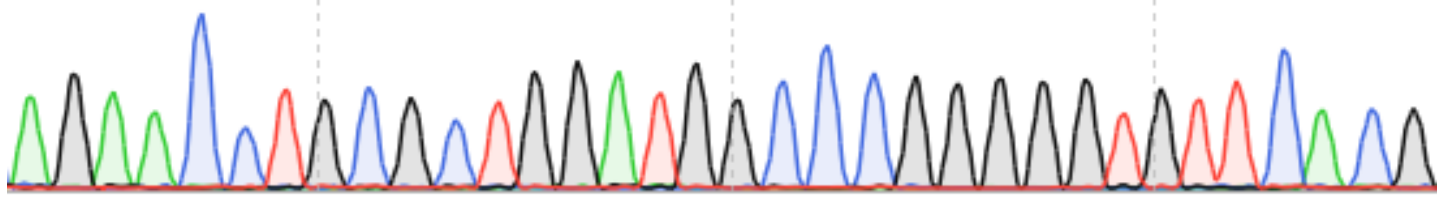

H. glaber exon 21

A T A T C C T C T C T G C T G A TGTGA G G T T G G A A T G C G T G T

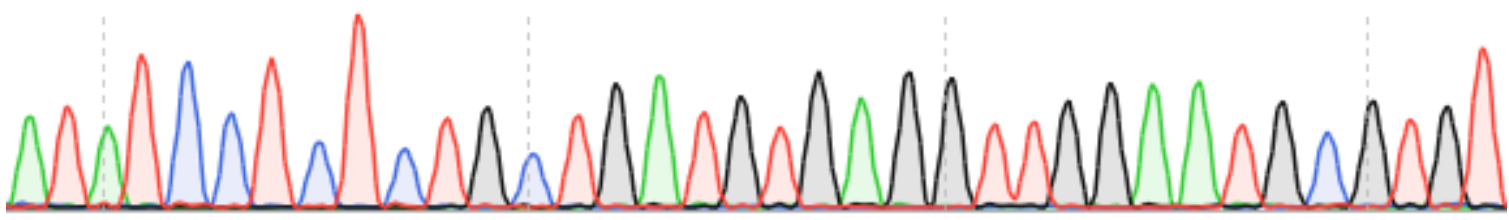




\section{Figure $\mathbf{S 2}$ continued}

\section{C}

ATGGGAAATTATAAATCTAGACCAACACAAACTTGTACTGATGAGTGGAAGAAGAAAGTCAATGAATCTT ATGTTATTACAATAGAAAGATCAGAAGATGACCCGAGGATCAAGGAAAAAGAATTTACAGAGATGAGACA TATTTTTGGCTCTGATGAAGCCTTCAGTAAAGTCAACCTAAATTACCGCACTGAAAGTGGACTATCTCTA CTGCATTTATGTTGCATTTGTGGAGGCAACCAGTCTCATATTCGAGCCCTTATGTTGAAAGGACTCTGCC CATCTCTACTGACAAGAAATGGATTCACAGCGTTGCACCTAGCAGTTTATAAGGACAGTGCAGAACTGAT CACTTCCTTCCTCCACAGTGGGGGTGATGTGCAGCAGGTGGGGTTCGGTGGCCTCACTGCACTCCACATT GCTGTCATCGCTGGTCACCTGGAGGCCCCTGATGTGTTACTCCAACACGGAGCTAATGTCAATGTTCAAG ATGCTGTTTTTTCACTCCATTGCACATTACAGTGTACTATGGGCATGAGCAGGTAACCCATCTCCTTTT GAAATTTGGTGCTGATGTAAATGTAAGTGGTGAAGTTGGGGATAGGCCTCTCCATCTGGCATCTGCAAAG GGATTCTTCAACATTGCAAAACTCCTGATGGAAGATGGGAATAAAGCAGATGTGAATGCTCAGGATAATG AAGACCATGACCCACTGCATTTCTGTTCTCATTTTGGCCATCACGAGATAATGAAGTATCTGCTCCAAAG TGACTCGGAAGTTCAGCCTCATGTTGTTAATATCTATGAAGACACTCCTTTGCACTTGGCATGCTACAAT GGCAAATTTGAAGTTGCTAAGGAGATCATTCAAATAACTGGAACAGAAAGTTTGACTAAGGAAAACATCT TTAGTGAGACAGCTTTTCACAGTGCTTGTACCTATGGCAAGAGCATTGATCTGGTTAAATTTCTTCTTGA TCAGAATGTGGTAAACATCAGTCACCAAGGAAGAGATGGGCACACAGGATTACACTCTGCTTGCTACCAT GGTCACATTCACCTGGTTCAGTTCTTACTGGACAATGGGGCTGATATGAATCTAGTTGCTTGTGATCCCA GCAGGTCTAGTGGTGAAAAAAATGAGCAGACATGTTTGATGTGGGCTTATGAAAAAGGACAGGATGCCAT TGTCACACTTCTGAAGCATTATAAGAGACCCCAGGATGAACTGCCCTGTAATGAATATTCTCGTCCTGGA GGAGATGGCTCCTATGTGTCTGTTCCATCCCCTTTGGGGAAGATTAAAAACATAACAAAAGAGAAGGTGG ATGTTCTCCTCCTGAGGGCCAGATTGCCTTCACATTTCCATCTTCAGCTCTCGGAAATTGAGTTCCATGA GATTATCGGCTCAGGTTCTTTTGGGAAAGTATACAAAGGACGATGCAGAAATAAAATAGTGGCTATAAAA CACTGTTGAGCCAACACCTATTGCTCCAAATCAGATATGGATATGTTTTGCCGAGAGGTGTCCATTCTCT GCCAGCTCAATCATCCCTGTGTCATTCAGTTTGTGGGCACTTGCTTAAATGATCCCAGCCAGTTTGCCAT TGTCACCCAGTACATCTCAGGAGGTTCTCTGTTCTCCCTCCTTCATGAGCAGAAAAGGGCCCTTGATTTG GAGTCTAAGTTAATTATTGCAGTAGATATTGCCAAAGGCATGGAGTACCTTCACCACCTGACCCAGCCAA TTATACACCGTGACTTGAACAGTCACAATATTCTTCTCTATGAGGACGGGCATGCTGTGGTGGCCGATTT TGGAGAATCAAGATTTCTACAATCTCTGGATGAAGACAACATGACAAAGCAACCTGGGAACCTGCGCTGG ATGGCCCGGGGGTGTTCACGCAGTGCGCCCGCTACACCACTAAGGCCGAAGTCTTCAGCTGCTCGCTGTG TCTGGGGGAGCTGCTGACTGGTGAGATTCCCTTCGCTCATCTCAAGCAGTGGCAGCGGCAGCAGACATGG CCTCCCACCACATCAGGCATCCCATTGGATATTCCATTCCCAAGCCCATATCCTCTCTGCTGATGTGAGG TTGGAATGCGTGTCCTGAAGGAAGACCTGAGTTTTCCAAAGTTGTTACCAAGTTAGAAGAATGTCTCTGC AACATTGAGCTGATGTCTCCGGCATCGAGTAACAGCAGTGGGTCTCTTTCACCTTCCTCTTCTTCTGATT GCCTGGCAAGCCAGGGAGCCCCTGGCCAGAGCACGTGGCAGCTTTACGGAGCCGTTTTGAGTTGGAGTGT GCTCTCAACGCAAGGTCCTATGCTGCCTGGCCCCAAAGCACTGGACCAAGTTCTCCTCAGGGGCTGTCTT TGGAGGAGATGTGCAGGAGTCTCCAGTGTTCCACCATCGACAAATACGGCTACATGTCTGATCCCCTGAG CCCCATGCATTTTCCTTCTTGCCGGGATAGTGGCAGCTTCAAGGACAGCAGCTGA

MGNYKSRPTQTCTDEWKKKVNESYVITIERSEDDPRIKEKEFTEMRHIFGSDEAFSKVNLNYRTESGLSL LHLCCICGGNQSHIRALMLKGLCPSLLTRNGFTALHLAVYKDSAELITSFLHSGGDVQQVGFGGLTALHI AVIAGHLEAPDVLLQHGANVNVQDAVFFTPLHITVYYGHEQVTHLLLKFGADVNVSGEVGDRPLHLASAK GFFN I AKLLMEDGNKADVNAQDNEDHDPLHFC SHFGHHE IMKYLLQSDSEVQPHVVNIYEDTPLHLACYN GKFEVAKEI IQITGTESLTKENIFSETAFHSACTYGKS I DLVKELLDQNVVNI SHQGRDGHTGLHSACYH GH IHLVQFLLDNGADMNLVACDPSRS SGEKNEQTCLMWAYEKGQDAIVTLLKHYKRPQDELPCNEYSRPG GDGSYVSVPSPLGKIKNITKEKVDVLLLRARLPSHFHLQLSEIEFHEI IGSGSFGKVYKGRCRNKIVAIK $\mathrm{HC}$ * 


\section{Figure $\mathbf{S 2}$ continued}

\section{D}

Identities: $431 / 490(88 \%)$

Positives: $453 / 490(92 \%)$

Gaps : $\quad 1 / 490(0 \%)$

Hgla 1 MGNYKSRPTQTCTDEWKKKVNESYVITIERSEDDPRIKEKEFTEMRHIFGSDEAFSKVNL 60 MGNYKSRPTQTC+DEWKKKV+ESY I IER EDD +IKE EF E+RHIFGSDEAFS+V+L

Mmu 1 MGNYKSRPTQTCSDEWKKKVSESYAIIIERLEDDLQIKENEFQELRHIFGSDEAFSEVSL

Hgla 61 NYRTESGLSLLHLCCICGGNQSHIRALMLKGLCPSLLTRNGFTALHLAVYKDSAELITSF NYRTE GLSLLHLCC CGGN+SHIRALMLKGL PS LTRNGF ALHLAVYKDS ELITS

Mmu 61 NYRTERGLSLLHLCCACGGNKSHIRALMLKGLRPSRLTRNGFPALHLAVYKDSLELITSL

Hgla 121 LHSGGDVQQVGFGGLTALHIAVIAGHLEAPDVLLQHGANVNVQDAVFFTPLHITVYYGHE LHSG DVQQ G+GGLTALHIA IAGH EA +VLLQHGANVNVQDAVFFTPLHI YYGHE Mmu 121 LHSGADVQQAGYGGLTALHIAAIAGHPEAVEVLLQHGANVNVQDAVFFTPLHIAAYYGHE

Hgla 181 QVTHLLLKFGADVNVSGEVGDRPLHLASAKGFFNIAKLLMEDGNKADVNAQDNEDHDPLH 240 QVT +LLKFGADVNVSGEVGDRPLHLASAKGFFNI KLL+E GNKADVNAQDNEDH PLH Mmu 181 QVTSVLLKFGADVNVSGEVGDRPLHLASAKGFFNIVKLLVE-GNKADVNAQDNEDHVPLH

Hgla 241 FCSHFGHHEIMKYLLQSDSEVQPHVVNIYEDTPLHLACYNGKFEVAKEIIQITGTESLTK FCS FGHH I+ YLLQSD EVQPHV+NIY DTPLHLACYNG FEVAKEI+ +TGTESLTK Mmu 240 FCSRFGHHNIVSYLLQSDLEVQPHVINIYGDTPLHLACYNGNFEVAKEIVHVTGTESLTK

ENIFSETAFHSACTYGKS I DLVKFLLDQNVVNI SHQGRDGHTGLHSACYHGH I HLVQFLL ENIFSETAFHSACTYGK+IDLVKFLLDQN VNI+H+GRDGHTGLHSACYHGHI LVQFLL

Hgla 361 DNGADMNLVACDPSRSSGEKNEQTCLMWAYEKGQDAIVTLLKHYKRPQDELPCNEYSRPG DNGADMNLVACDPSRSSGEK+EQTCLMWAYEKG DAIVTLLKHYKRPQDELPCNEYS+PG Mmu 360 DNGADMNLVACDPSRSSGEKDEQTCLMWAYEKGHDAIVTLLKHYKRPQDELPCNEYSQPG

Hgla 421 GDGSYVSVPSPLGKIKNITKEKVDVLLLRARLPSHFHLQLSEIEFHEIIGSGSFGKVYKG 480 GDGSYVSVPSPLGKIK++TKEK DVLLLRA LPS FHLQLSEIEFHEIIGSGSFGKVYKG Mmu 420 GDGSYVSVPSPLGKIKSMTKEKADVLLLRAELPSRFHLQLSEIEFHEIIGSGSFGKVYKG

Hgla 481 RCRNKIVAIK 490 RCRNKIVAIK

Mmu 480 RCRNKIVAIK 489 


\section{Figure S2 continued}

$\mathbf{E}$

ATGGGAAATTATAAATCTAGACCAACACAAACTTGTACTGATGAGTGGAAGAAGAAAGTCAATGAATCTT ATGTTATTACAATAGAAAGATCAGAAGATGACCCGAGGATCAAGGAAAAAGAATTTACAGAGATGAGACA TATTTTTGGGCCCCTGATGTGTTACTCCAACACGGAGCTAATGTCAGTGTTCAAGATGCTGTTTTTTTCA CTCCATTGCACATTACAGTGTACTATGGGCATGAGCAGGTAACCCATCTCCTTTTGAAATTTGGTGCTGA TGTAAATGTAAGTGGTGAAGTTGGGGATAGGCCTCTCCATCTGGCATCTGCAAAGGGATTCTTCAACATT GCAAAACTCCTGATGGAAGATGGGAATAAAGCAGATGTGAATGCTCAGGATAATGAAGACCATGACCCAC TGCATTTCTGTTCTCATTTTGGCCATCACGAGATAATGAAGTATCTGCTCCAAAGTGACTCGGAAGTTCA GCCTCATGTTGTTAATATCTATGAAGACACTCCTTTGCACTTGGCATGCTACAATGGCAAATTTGAAGTT GCTAAGGAGATCATTCAAATAACTGGAACAGAAAGTTTGACTAAGGAAAACATCTTTAGTGAGACAGCTT TTCACAGTGCTTGTACCTATGGCAAGAGCATTGATCTGGTTAAATTTCTTCTTGATCAGAATGTGGTAAA CATCAGTCACCAAGGAAGAGATGGGCACACAGGATTACACTCTGCTTGCTACCATGGTCACATTCACCTG GTTCAGTTCTTACTGGACAATGGGGCTGATATGAATCTAGTTGCTTGTGATCCCAGCAGGTCTAGTGGTG AAAAAAATGAGCAGACATGTTTGATGTGGGCTTATGAAAAAGGACAGGATGCCATTGTCACACTTCTGAA GCATTATAAGAGACCCCAGGATGAACTGCCCTGTAATGAATATTCTCGTCCTGGAGGAGGTGCCCACTTT TGCATTCACTTTTCATTGTTGTGATGCATGGCACCTAAAGAGAAGGTGGATGTTCTCCTCCTGAGGGCCA GATTGCCTTCACATTTCCATCTTCAGCTCTCGGAAATTGAGTTCCATGAGATTATCGGCTCAGGTAACCT AAAACACCACAGGTCTGCTTTAGTTTCATGAACCCGAGATAATCTGTGTTTATTTATTTTAAACAATTTC AGGTTCTTTTGGGAAAGTATACAAAGGACGATGCAGAAATAAAATAGTGGCTATAAAACACTGTTGA

MGNYKSRPTQTCTDEWKKKVNESYVITIERSEDDPRIKEKEFTEMRHIFGPLMCYSNTELMSVFKMLFFS LHCTLQCTMGMSR* 


\section{Figure $\mathbf{S 2}$ continued}

$\mathbf{F}$

Exon 5

$\begin{array}{lllllllllllllllll}D & S & \text { L } & \text { E } & \text { L } & \text { I } & \text { T } & \text { S } & \text { I } & \text { L } & \text { H } & \text { S } & G & \text { A } & \text { D } & \text { V } & Q\end{array}$

Mmu ...agGATAGCCTGGAACTTATCACTTCACTGTTGCATAGCGGAGCAGATGTTCAG

Fdam . . . agGA-AGTGCAGAATCGATCACTTCCCTGCTGCACAGTGGGACTGATGAGCAG

Hgla . . agGACAGTGCAGAACTGATCACTTCCTTCCTCCACAGTGGGGGTGATGTGCAG

$\begin{array}{lllllllllllllllll}D & \text { S } & \text { A } & \text { E } & \text { L } & \text { I } & \text { T } & \text { S } & \text { F } & \text { L } & \text { H } & \text { S } & \text { G } & \text { G } & \text { D } & \text { V } & \text { Q }\end{array}$

$\mathrm{Mmu}$

$\begin{array}{llllllllllllllll}\mathbf{Q} & \mathbf{A} & \mathrm{G} & \text { Y } & \text { G } & \text { G } & \text { L } & \text { T } & \text { A } & \text { L } & \text { H } & \text { I } & \text { A } & \text { A } & \text { I } & \text { A }\end{array}$

Fdam CAAGCGGGATATGGCGGCCTCACAGCCCTCCACATCGCTGCAATAGCT . . .

Hgla CAGGTGGGGTAAGGTGGCTTCTCTGCGCTCCACACTGCTGTCATAGCC . . . CAGGTGGGGTTCGGTGGCCTCACTGCACTCCACATTGCTGTCATCGCT . . .

$$
\begin{array}{llllllllllllllll}
\text { Q } & \text { V } & \text { G } & \text { F } & \text { G } & \text { G } & \text { L } & \text { T } & \text { A } & \text { L } & \text { H } & \text { I } & \text { A } & \text { V } & \text { I } & \text { A }
\end{array}
$$

F. damarensis exon 5

A G G A A G T G C A G A A T C G

C $A G G T G G G G T A A G G T G G C$
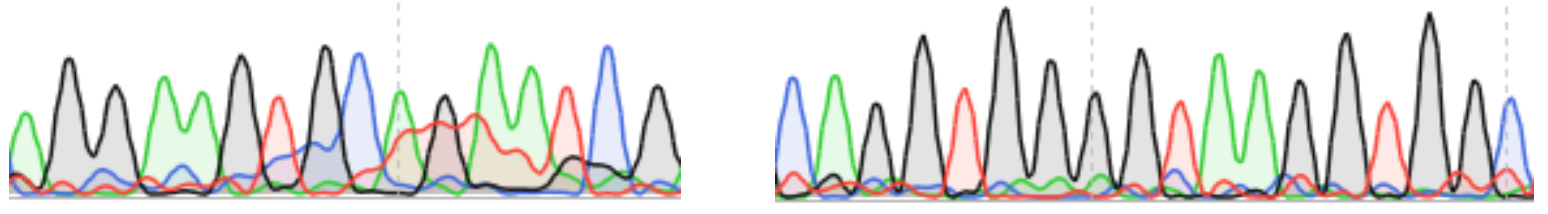

Exon 15

$\begin{array}{llllllllllllllllll}\mathbf{S} & \mathbf{F} & \mathrm{G} & \mathrm{K} & \mathrm{V} & \mathrm{Y} & \mathrm{K} & \mathrm{G} & \mathrm{R} & \mathrm{C} & \mathrm{R} & \mathrm{N} & \mathrm{K} & \mathrm{I} & \mathrm{V} & \mathrm{A} & \mathrm{I}\end{array}$ Mmu ...agGTTCTTTTGgG AAAGTCTATAAAGGGCGATGCAGAAATAAAATAGTGGCAATC... Fdam. . . agGTTCTTTAGGGGAAGGTAAATAAAGGATGATGCAGGAATAAGATAGCGGCTATA . . Hgla...agGTTCTTTTGGG AAAGTATACAAAGGACGATGCAGAAATAAAATAGTGGCTATA...

$$
\begin{array}{lllllllllllllllll}
S & F & G & K & V & Y & K & G & R & C & R & N & K & I & V & A & I
\end{array}
$$

F. damarensis exon 15

$A G G T T C T T T A G G G G A A G G T A A A T A A A G G A T G A T G C A G G$

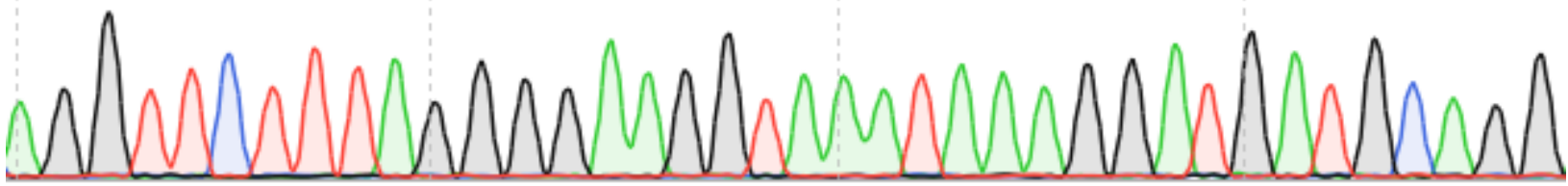

